\title{
Survey on Performance of Free Space Optical Communication Links under Various Field Parameters
}

\author{
Priyanka Jangir ${ }^{1}$, Bhuperdra Suman ${ }^{2}$, Rashmi Chaudhary ${ }^{3}$ \\ ${ }^{1}$ (ECE,DIT/ Uttrakhand Technical University ,India) \\ ${ }^{2}$ (Defense Electronics Application Laboratory, DRDO, India) \\ ${ }^{3}$ (ECE,DIT/ Uttrakhand Technical University ,India)
}

\begin{abstract}
Free space optics is a line-of-sight technology, which uses LASERS and Photo detectors to provide optical connections between two points-without the fiber. Increased needs for bandwidth require technology that goes beyond traditional copper lines. The technology advancement in internet scales faster than Moore's law which makes it infeasible to dig installed lines in metropolitan areas; the industry now realized the benefits of free space optical communication. Free-space optical (FSO) communication links are attractive because they provide a means of low cost, high bandwidth, reduced time-to-market, secure and are a reliable means of communications. This paper describes the benefits of FSO along with operation of such links working outdoors in an open atmosphere under various effecting field parameters such as atmospheric attenuation, beam divergence and scintillation where the performance of FSO link is evaluated in terms of transmitted and received power.
\end{abstract}

Keywords: Bit error, rate channel capacity, free space optical communication, link performance

\section{Introduction}

The astonishing growth of internet traffic over the last decade by the general public has caused the phenomenon that is referred to as the "last mile bottleneck." The "last mile bottleneck" is the shortage in bandwidth that is a result of the connectivity technologies in metropolitan areas being unable to keep up with the increasing needs. Willebrand [1] state that internet traffic has scaled faster than Moore's law, which is the exponential growth in technology. Free-space optical communication (FSO) systems (in space and inside the atmosphere) have developed in response to a growing need for high-speed and tap-proof communication systems. Links involving satellites, deep-space probes, ground stations, unmanned aerial vehicles (UAVs), high altitude platforms (HAPs), aircraft, and other nomadic communication partners are of practical interest. Moreover, all links can be used in both military and civilian contexts. FSO is the next frontier for net-centric connectivity, as bandwidth, spectrum and security issues favor its adoption as an adjunct to radio frequency (RF) communications [2].

While fixed FSO links between buildings have long been established and today form a separate commercial product segment in local and metropolitan area networks [3], the mobile and long-range applications of this technology are aggravated by extreme requirements for pointing and tracking accuracy because of the small optical beam divergences involved. This challenge has to be addressed to fully exploit the benefits of optical links. Furthermore, long-haul optical links through the atmosphere suffer from strong fading as a result of index-of-refraction turbulence (IRT) and link blockage by obscuration such as clouds, snow and rain [4].

\section{Faso Communication System}

A typical FSO transmitter (LASER, VCSEL or LED) forms a cone shaped volume in 3 dimensions in which a potential receiver equipped with a photo detector can receive the signal. The exact shape of this cone is determined by the transmission power (for range) and divergence angle. A LASER has the smallest (in micro radian range) and an LED has the widest (a few hundred milli radians) divergence angle of the three types of transmitters. FSO can operate in large swathes of unlicensed spectrum reaching speeds up to $1 \mathrm{Gbps}$. Additionally, FSO transceivers have much smaller form factors, are less power-consuming (100 microwatts for $10-100 \mathrm{Mbps}$ ), very reliable (lifetime of more than 10 years), cheap and offer highly directional beams for spatial reuse and security. The block diagram of fso link as: 


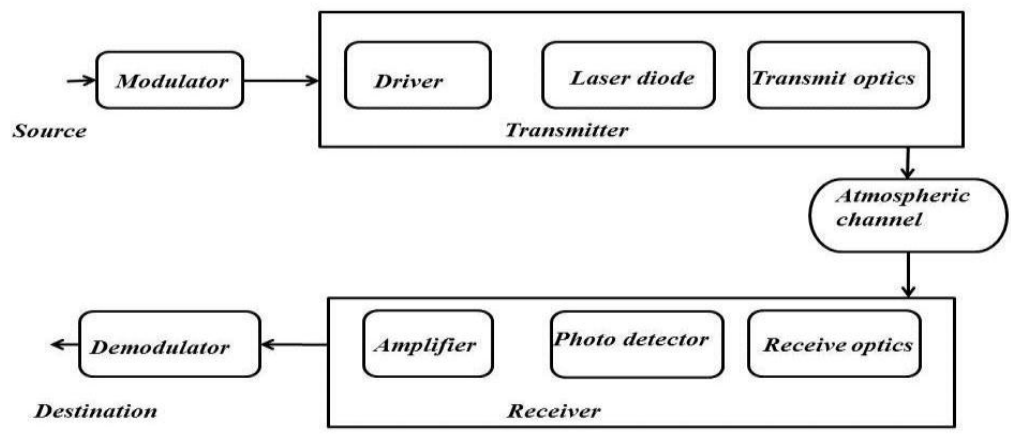

Fig 1: Block diagram of FSO communication system.

\section{Theory Of Optical Link Performance}

The FSO link performances can be determined by several parameters including geometrical loss, link margin, received power and BER. There are two parameters to evaluate the FSO link performances which are the received power and BER. Theoretically, the basic communication principle stated that received power must be less than transmitted power, $\mathrm{PR} \leq \mathrm{PT}$, according to[5] :

$$
P_{R}=P_{T} \text {-Total losses }
$$

Where $P_{R}(\mathrm{dBm})$ is the received power, $P_{T}(\mathrm{dBm})$ is the transmitted power. According to [5], total losses in a FSO communication system would cover all the losses caused by the atmospheric phenomena, $L_{\text {ATM }}(\mathrm{dB})$ which can be calculated as in Eq. (5), geometrical loss, $L_{G E O}(\mathrm{~dB})$ and system loss, $L_{S Y S}(\mathrm{~dB})$. Therefore, the new equation for FSO received power is as in Eq. (2):

$$
P_{R}=P_{\text {Tcomb }}-L_{A T M}-L_{G E O}-L_{S Y S}
$$

The total transmitted power can be obtained in Eq. (3):

$$
P_{\text {Tcomb }}=P_{T}+10 \log _{10}\left(N_{T}\right)
$$

Where, $N_{T}$ is the number of transmitter lenses on a single FSO unit. Geometrical loss and system loss are the internal losses occurred within the FSO transceiver. Both losses are fixed on all FSO link and cannot be neglected. $L_{S Y S}$ Is manufacturer defined; meanwhile in [5] $L_{G E O}$ can be calculated as in Eq. (4):

$$
L_{\text {GEO }}=-10 \log _{10}\left[\frac{A_{\text {RTOtal }}}{\pi\left[\left([\theta)^{2}\right.\right.}\right]
$$

$\ell(\mathrm{km})$ is the distance of the optical path where the laser beams travel and $\theta(\mathrm{mrad})$ is the divergence angle which is the angle of the cone of light emitted from the transmitter. Meanwhile, $\left(m^{2}\right) A_{R T o t a l}\left(m^{2}\right)$ is the total area of the receiver apertures on a single FSO unit.

According to Beers-Lambert Law, the atmospheric losses for any laser power is in a form of exponential equation of:

$$
L_{\text {ATM }}=e^{\text {N }}
$$

Where $\ell(\mathrm{km})$ is the transmittance range of the laser and $\sigma$ is the typical attenuation coefficients (0.1 for clear air)[6].

The robustness of the design of any optical communication system can be effectively verified by critically applying performance checks on the system. The evaluation criteria should provide a precise determination and separation of dominant system limitations, making them crucial for the suppression of propagation disturbances and performance improvement. The Bit Error Rate (BER) of an optical link is the most important measure of the faithfulness of the link in transporting the binary data from transmitter to receiver. The BER quantifies the rate of errors and is defined as the probability of an error occurring per transported bit. The bit error rate takes the simple form

$$
\mathrm{BER}=\frac{1}{2} \operatorname{erfc}\left(\frac{Q}{\sqrt{2}}\right)
$$

Where $\mathrm{Q}$ is quality factor and 'erfc' denotes the complementary error function [7].

\subsection{Atmospheric channel attenuation}

\section{Parameters Effecting Fso Link}

The atmospheric channel attenuates the field traversing it as result of absorption and scattering processes. The concentrations of matter in the atmosphere, which result in the signal attenuation vary spatially and temporally, and will depend on the current local weather conditions. For a terrestrial FSO link transmitting optical signal through the atmosphere, the received irradiance at a distance, L from the transmitter is related to the transmitted irradiance by the Beer-Lambert's law given as [8]: 


$$
\tau(\lambda, \mathrm{L})=\frac{P_{R}}{P_{J}}=\exp (-\Upsilon(\lambda), \mathrm{L}),
$$

where $\Upsilon(\lambda)$ and $P_{R}$ represent the total attenuation/extinction coefficient $\left(\mathrm{m}^{-1}\right)$ and the received optical power at a distance, L. $P_{T}$ and, $\tau(\lambda, \mathrm{L})$ represent the optical power at the optical source and the transmittance of the atmosphere at wavelength, $\lambda$ respectively. The attenuation of the optical signal in the atmosphere is due to the presence of molecular constituents (gases) and aerosol. The aerosol is made up of tiny particles of various shapes ranging from spherical to irregular shapes suspended in the atmosphere. The particles generally have sizes spanning from sub micrometer to a few tens of centimeters. Hence, the attenuation coefficient is the sum of the absorption and the scattering coefficients from aerosols and molecular constituents of the atmosphere[9]. It follows therefore that:

$$
\Upsilon(\lambda)=\alpha_{m}(\lambda)+\alpha_{a}(\lambda)+\beta_{m}(\lambda)+\beta_{a}(\lambda),
$$

The first two terms represent the molecular and aerosol absorption coefficients, respectively while the last two terms are the molecular and aerosol scattering coefficients respectively. Absorption takes place when there is an interaction between the propagating photons and molecules (present in the atmosphere) along its path. Absorption is wavelength dependent and therefore selective. This leads to the atmosphere having transparent zones-range of wavelengths with minimal absorptions-referred to as the transmission windows. However, the wavelengths used in FSO are basically chosen to coincide with the atmospheric transmission windows [10], resulting in the attenuation coefficient being dominated by scattering. The attenuation is thus reduced to:

$$
\Upsilon(\lambda) \cong \beta_{\alpha}(\lambda),
$$

TABLE1: Typical atmospheric scattering particles with their radii and scattering process at $\lambda=850 \mathrm{~nm}$

\begin{tabular}{|l|l|l|l|}
\hline Type & Radius $(\mu \mathrm{m})$ & Size parameter & Scattering parameter \\
\hline Air molecules & 0.0001 & 0.00074 & Rayleigh \\
\hline Haze particle & $0.01-1$ & $0.074-7.4$ & Rayleigh-Mie \\
\hline Fog droplet & $1-20$ & $7.4-147.8$ & Mie-Geometrical \\
\hline Rain & $100-10000$ & $740-74000$ & Geometrical \\
\hline snow & $1000-5000$ & $7400-37000$ & Geometrical \\
\hline hail & $5000-50000$ & $37000-370000$ & Geometrical \\
\hline
\end{tabular}

The Mie scattering occurs when the particle size is comparable to the beam size. Geometrical loss counts for the losses that occur due to the divergence of the optical beam.

\subsection{Scintillation}

Atmospheric scintillation, as used in this document, can be thought of as changing light intensities in time and space at the plane of a receiver detecting the signal from a transmitter at a distance. The received signal level at the detector fluctuates due to thermally induced changes in the index of refraction of the air along the transmit path. The index changes cause the atmosphere to act like a collection of small prisms and lenses that deflect the light beam into and out of the transmit path. The time scale of these fluctuations is about the time it takes a volume of air the size of the beam to move across the path and therefore is related to wind speed. For the case of free-space optics, which implies horizontal path propagation and therefore stronger scintillation, the distribution tends to be more exponential. One parameter that is often used as a measure of the scintillation strength is the atmospheric structure parameter $\operatorname{or}_{n}{ }^{2}$. This parameter, which is directly related to wind speed, roughly measures how turbulent the atmosphere is.

\subsection{Beam divergence}

One of the primary advantages of FSO transmission is the narrowness of the transmitted laser beam that can be achieved with well-designed optics [11]. This narrow beam allows for secure and efficient transmission with a major fraction of the transmitted power being collected by the receiver. Typically, the optical beam width from a FSO transceiver will be relatively wide (2-10-mrad divergence, which is equivalent to a beam spread of $2-10 \mathrm{~m}$ at $1 \mathrm{~km}$ ), as is generally the case in non-tracking applications. For such applications, the system must compensate for any platform motion by having a beam width and total FOV (TFOV) that is larger than either transceiver's anticipated platform motion.

\subsection{Link margin and attenuation versus range}

The relation between the link margin and attenuation versus range [11] is shown. The first curve shows how much margin a given system has at a given range to compensate for both scattering and scintillation losses. This second curve loses accuracy at low attenuation $(<30 \mathrm{~dB} / \mathrm{km})$ because it does not take into account scintillation losses. 


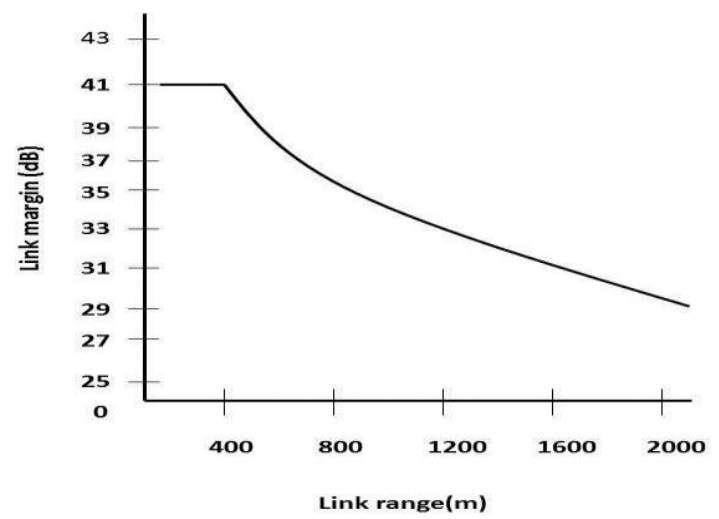

Fig3. Link margin versus range

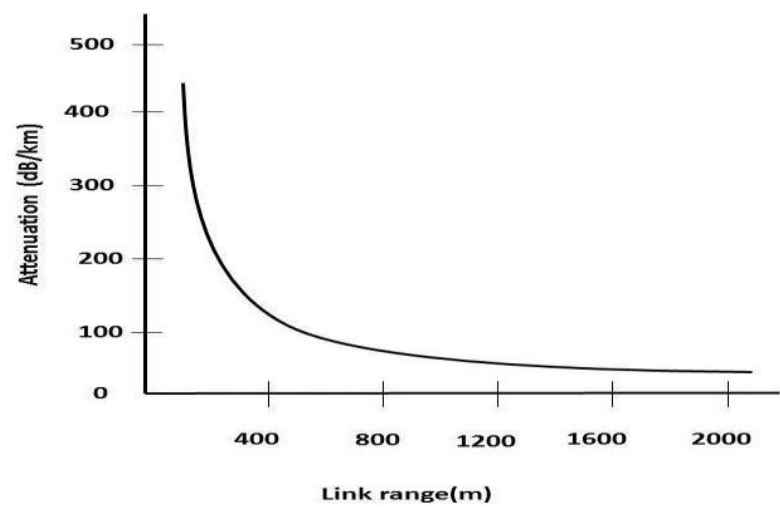

Fig4. Attenuation versus range

\subsection{Benefits}

\section{Advantages And Drawbacks}

The attraction of FSO is its high data transmission rate and its exemption from spectrum regulation. The latter is especially significant for military ground forces setting up camps and forward operating bases overseas. At the very least it is time consuming. To be able to circumvent the spectrum management bureaucracy is a huge advantage given urgent communication requirements. Since light beams do not interfere with each other as long as they are not coaxial, commanders need not be concerned with electromagnetic compatibility problems. FSO is as ready a resource as a light bulb in a socket, and installation of FSO equipment is quick and inexpensive.

\subsection{Drawbacks or challenges of the technology}

5.2.1. Laser Eye Safety. It is important to keep in mind, especially if FSO is to gain widespread use for camp communications, that lasers must be operated within certain levels of irradiance $\left[\mathrm{w} / \mathrm{m}^{2}\right.$ ] for eye safety. The harmful level of exposure is a function of wavelength and is tabulated in American National Standards Institute (ANSI) Standard.

5.2.2 Disruption by weather. Although FSO may at times be capable of greater range, its greater susceptibility to degradation from incidents of heavy fog or dust will drive down its attainable availability figures. This will depend on which region of the world FSO is planned for. For example, frequent dust storms of such severity as to result in black out conditions often occur in tactical desert conditions. Furthermore, the summer heat in the desert and along coastlines induces extreme refractive turbulence that would cause optical defocusing and beam wander [12].

\section{Conclusion}

The applications that FSO technology is more efficient for clear weather, short distance link establishment, such as last-mile connections to broadband network backbones, and backbone links between buildings in a MAN or CAN environment. The advantages of FSO are due to the characteristics of a laser beam, especially from its high frequency, coherency and low divergence, which lead to 
efficient delivery of power to a receiver and a high information-carrying capacity. The main problems of FSO links working outdoors in the atmosphere result from attenuation and fluctuation of optical signal at a receiver as predicting the free space channel is always a difficult task. To improve reliability, a number of new methods can be used. After considering all its advantages and disadvantages it is clear that FSO has good prospects for widespread implementation. FSO technology is ready for utilization as terrestrial links, mobile links and satellite links.

\section{Acknowledgements}

The authors acknowledge the facility provided by the Director (DEAL, Dehradun) as well as encouragement and support given by Dr Sandeep vijay, Professor and Head (ECE and AEI Dept), DIT University, Dehradun.

\section{References}

[1]. H. Willebrand, Free-space optics: enabling optical connectivity in today's networks, Indianapolis, IN: Sams Publishing, 2002.

[2]. Das, S., Henniger, H., Epple, B., Moore, C., Rabinovich,W., Sova, R., Young, D. Requirements and challenges for tactical freespace laser comm . In IEEE Military Communications Conference. San Diego (USA), 2008, p. 1 - 10.

[3]. H. Willebrand., Ghuman, B. Fiber optics without fiber. IEEE Spectrum, 2001, vol. 38, no. 8, p. 40- 45.

[4]. H. Henniger, O. Wilfert. An introduction to free-space optical communications. radioengineering, Vol. 19, No. 2, June 2010, P.203-212.

[5]. I. D. A. Singapore, A trial-based study of free-space optics systems in Singapore, Info-Communications Development Authority of Singapore (Ida), Singapore 2002.

[6]. N. Haedzerin Md Noor, Ahmed Wathik Naji And Wajdi Al-Khateeb, Performance analysis of a free space optics link with multiple transmitters/receivers, lium Engineering Journal, Vol. 13 No. 1, 2012 Pg.49-58.

[7]. G. Soni, Jagjit M. Singh, Impact of Beam Divergence on the Performance of Free Space Optical System, IJSRP, Volume 2, Issue 2, February 2012, ISSN 22503153

[8]. R. M. Gagliardi and S. Karp, Optical communications, 2nd edition New York: John Wiley, 1995.

[9]. H. Hemmati, Deep space optical communications, in Deep space communications and navigation series California, 2005

[10]. S. Bloom, E. Korevaar, J. Schuster, and H. Willebrand, "Understanding the performance of free-space optics", Journal of optical Networking, vol. 2, June 2003 pp. 178-200.

[11]. S. Bloom, E. Korevaar, J. Schuster, and H. Willebrand, Understanding the performance of free-space optics, Journal of optical Networking, vol. 2, June 2003, pp. 178-200.

[12]. S. Asif \& P. K. Yadav An overview to free space optics and its advantage over fiber optics International Journal of Electronics and Communication Engineering (IJECE) Vol.1, Issue 1 Aug 2012 pg. 13-22. 